\title{
Randomized controlled study comparing 2 surgical techniques for decompressive craniectomy: with watertight duraplasty and without watertight duraplasty
}

\author{
Eduardo Vieira, MD, Thiago C. Guimarães, MD, Igor V. Faquini, MD, Jose L. Silva Jr., MD, \\ Tammy Saboia, MD, Rodrigo V. C. L. Andrade, MD, Thaís L. Gemir, MD, Valesca C. Neri, MD, \\ Nivaldo S. Almeida, MD, and Hildo R. C. Azevedo-Filho, MSc, PhD, FRCS \\ Department of Neurological Surgery, Hospital da Restauração, Recife, Brazil
}

OBJECTIVE Decompressive craniectomy (DC) is a widely used procedure in neurosurgery; however, few studies focus on the best surgical technique for the procedure. The authors' objective was to conduct a prospective randomized controlled trial comparing 2 techniques for performing DC: with watertight duraplasty and without watertight duraplasty (rapid-closure DC).

METHODS The study population comprised patients ranging in age from 18 to 60 years who were admitted to the Neurotrauma Service of the Hospital da Restauração with a clinical indication for unilateral decompressive craniectomy. Patients were randomized by numbered envelopes into 2 groups: with watertight duraplasty (control group) and without watertight duraplasty (test group). After unilateral DC was completed, watertight duraplasty was performed in the control group, while in the test group, no watertight duraplasty was performed and the exposed parenchyma was covered with Surgicel and the remaining dura mater. Patients were then monitored daily from the date of surgery until hospital discharge or death. The primary end point was the incidence of surgical complications (CSF leak, wound infection, brain abscess, or subgaleal fluid collections). The following were analyzed as secondary end points: clinical outcome (analyzed using the Glasgow Outcome Scale [GOS]), surgical time, and hospital costs.

RESULTS Fifty-eight patients were enrolled, 29 in each group. Three patients were excluded, leaving 27 in the test group and 28 in the control group. There were no significant differences between groups regarding age, Glasgow Coma Scale score at the time of surgery, GOS score, and number of postoperative follow-up days. There were 9 surgical complications ( 5 in the control group and 4 in the test group), with no significant differences between the groups. The mean surgical time in the control group was 132 minutes, while in the test group the average surgical time was 101 minutes, a difference of 31 minutes $(p=0.001)$. The mean reduction in total cost was $\$ 420.00$ USD (a $23.4 \%$ reduction) per procedure in the test group.

CONCLUSIONS Rapid-closure DC without watertight duraplasty is a safe procedure. It is not associated with a higher incidence of surgical complications (CSF leak, wound infection, brain abscess, or subgaleal fluid collections), and it decreased surgical time by 31 minutes on average. There was also a hospital cost reduction of \$420.00 USD (23.4\% reduction) per procedure.

Clinical trial registration no.: NCT02594137 (clinicaltrials.gov)

https://thejns.org/doi/abs/10.3171/2017.4.JNS152954

KEY WORDS decompressive craniectomy; traumatic brain injury; brain edema; duraplasty

$\mathrm{D}$ ECOMPRESSIVE craniectomy (DC) is very effective in the treatment of refractory intracranial hypertension and is a widely used procedure in neurosurgical practice. In the medical literature, however, there are few well-designed studies focusing on the best surgical technique for the procedure; most studies are retrospective series and expert opinion (Levels 4 and 5). This generates debate and controversy about the best technique to be used, especially regarding how to handle the opened dura mater. In 2011, Güresir et al. ${ }^{5}$ published a series of cases introducing the concept of rapid-closure DC, in which, after opening the dura mater, no watertight duraplasty is

ABBREVIATIONS DC = decompressive craniectomy; GCS = Glasgow Coma Scale; GOS = Glasgow Outcome Scale; TBI = traumatic brain injury .

SUBMITTED December 17, 2015. ACCEPTED April 18, 2017.

INCLUDE WHEN CITING Published online November 17, 2017; DOI: 10.3171/2017.4.JNS152954. 


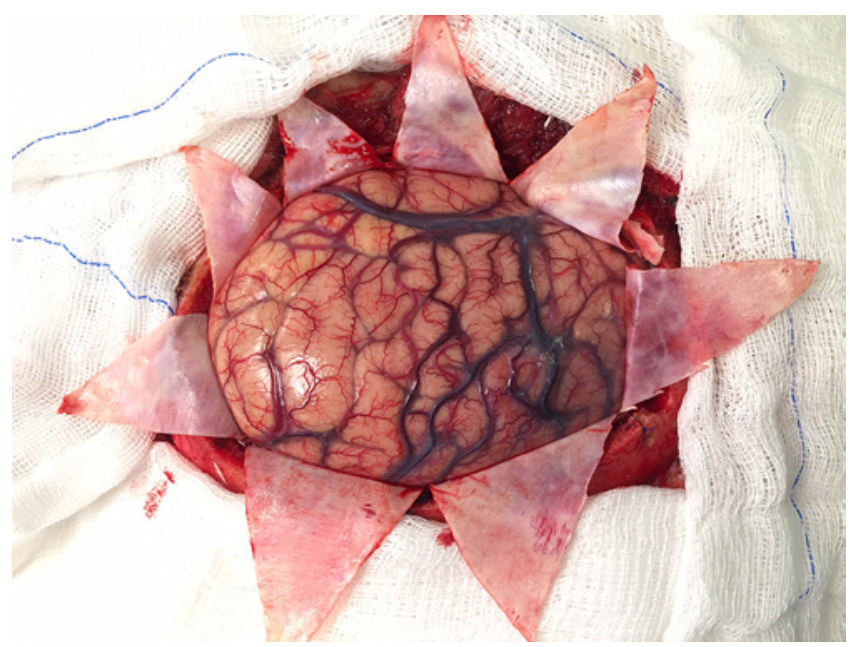

FIG. 1. Intraoperative photograph showing a craniotomy (at least $12 \times 15$ $\mathrm{cm}$ ) and removal of the temporal bone until flush with the middle fossa floor. Figure is available in color online only.

performed and the exposed brain parenchyma is covered by the remaining opened dura and Surgicel (Ethicon). Our objective was to conduct a prospective randomized controlled study comparing 2 surgical techniques for DC: with watertight duraplasty and without watertight duraplasty (rapid-closure DC).

\section{Methods \\ Patient Population}

Institutional review board approval was obtained for a single-center randomized controlled prospective trial. The study population comprised patients ranging in age from 18 to 60 years old who were admitted to the Neurotrauma Service of the Hospital da Restauração from January 2012 to December 2013 to undergo unilateral DC. Patients with intraaxial contusions or hematomas requiring surgical evacuation, in which case injury to the arachnoid mater could lead to an increased risk of CSF leak, were excluded. Patients included in the study had written informed consent obtained from next of kin or guardian as appropriate. This study was registered with clinicaltrials.gov (registration no. NCT02594137).

\section{Study Intervention and Surgical Technique}

After clinical indication for unilateral DC, patients were randomized by numbered envelopes into 2 groups: with watertight duraplasty (control group) and without watertight duraplasty (rapid-closure DC) (test group). In all cases, a large trauma flap (i.e., large reverse question mark starting from the tragus and extending to the midline) was performed. The skin, galea, and muscle layers were elevated according to the surgeon's preference. Thus, a wide (at least $12 \times 15 \mathrm{~cm}$ ) craniotomy was performed, and the temporal bone was removed until it was flush with the middle fossa floor (Fig. 1). After dural opening, watertight duraplasty with pericranium or an artificial graft (at the surgeon's discretion) was performed in the control group (Fig. 2), while no watertight duraplasty was performed in

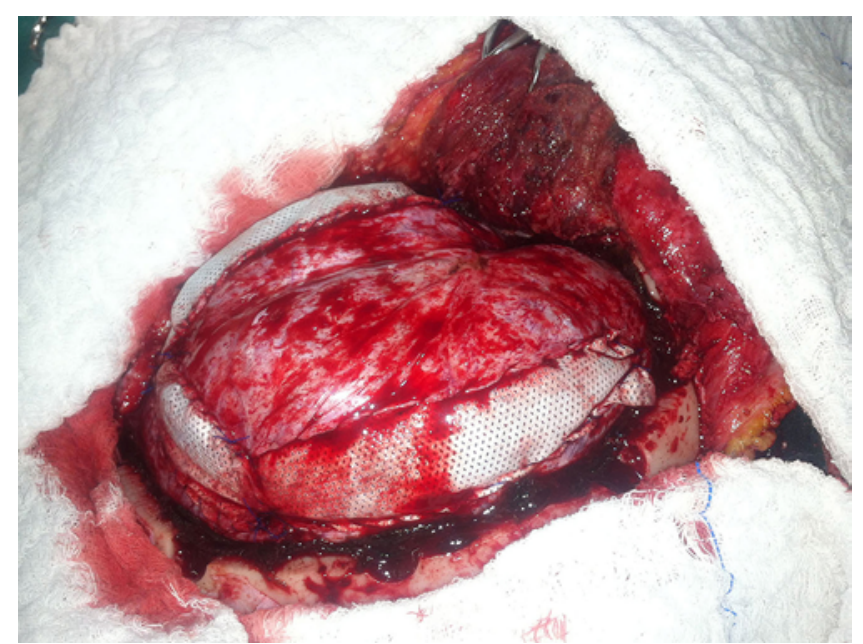

FIG. 2. Intraoperative photograph showing a watertight duraplasty that was performed in patients randomized to the control group. Figure is available in color online only.

the test group, in which the exposed brain parenchyma was covered with Surgicel (Fig. 3). Usual closure was then performed. Patients were then monitored daily by evaluators blinded to the randomization (control or test group) from the date of surgery until hospital discharge or death.

The primary end point of our study was the incidence of complications, namely CSF leak (CSF drainage through surgical wound), subgaleal fluid collections (CSF drainage to the subcutaneous/epidural space, but not through surgical wound), wound infection (limited to the subcutaneous/ epidural space; not involving the brain parenchyma), or brain abscess (infection involving the brain parenchyma). Cranial CT scans were obtained routinely between the 7th and 10th postoperative days to evaluate the occurrence of subgaleal fluid collections. The following were analyzed as secondary end points: clinical outcome (analyzed using the Glasgow Outcome Scale [GOS]), surgical time, and

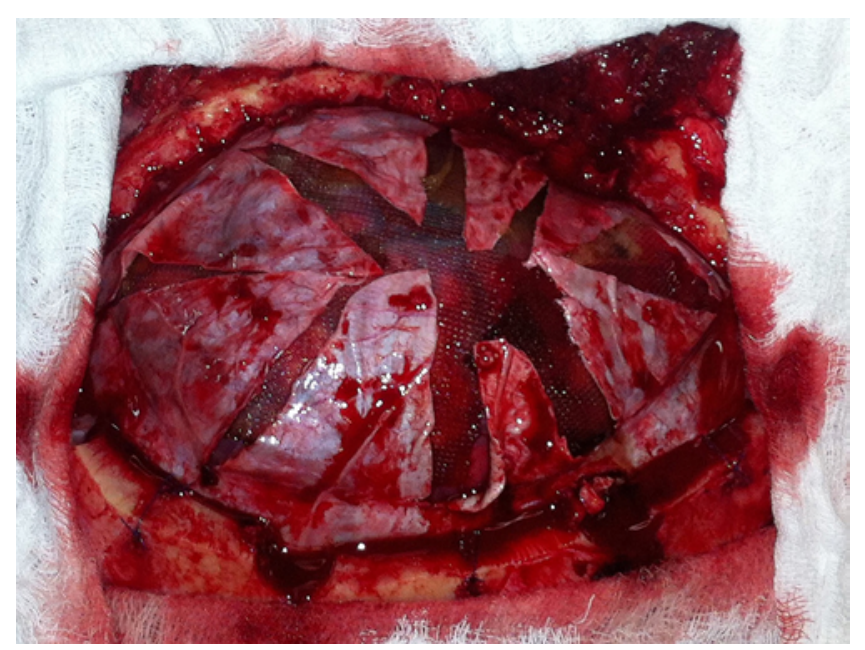

FIG. 3. Intraoperative photograph showing a rapid-closure DC without watertight duraplasty performed in patients randomized to the test group. Figure is available in color online only. 
TABLE 1. Etiology leading to the indication of decompressive craniectomy

\begin{tabular}{lc}
\hline \multicolumn{1}{c}{ Etiology } & No. of Patients \\
\hline TBI & 36 \\
\hline MCA infarction & 15 \\
\hline Vasospasm due to subarachnoid hemorrhage & 5 \\
\hline Dural venous sinus thrombosis & 2 \\
\hline
\end{tabular}

$\mathrm{MCA}=$ middle cerebral artery.

procedure costs. To estimate procedure costs, the mean costs of a DC procedure per minute were calculated based on anesthetic drug usage and operating room utilization time (data supplied by hospital administration). Costs analysis further included cost of artificial dural grafts (when used).

\section{Statistical Analysis}

For categorical variables we used the Pearson chisquare test or Fisher's exact test if the chi-square test was not applicable; the Student t-test or Mann-Whitney test was used for numeric variables. Based on previous studies, we found that the surgical mean time for decompressive DC with watertight dural closure is 129 minutes. For our study, we assumed that the use of rapid-closure DC without watertight duraplasty would reduce surgical time by $40 \%$. Using a 2 -sided alpha of $0.01,25$ patients per treatment group would give a $99 \%$ power to detect at least a $40 \%$ decrease in surgical time.

\section{Results}

Fifty-eight patients with a mean age of 33.4 years (1859 years) were included. Thirty-five patients were male. The main indication for DC was traumatic brain injury (TBI) (Table 1). The mean Glasgow Coma Scale (GCS) score at the time of surgery was 8.6 points. Overall, 19 patients presented with signs of herniation that manifested as anisocoria. The mean GOS score for the study population was 2.85 .

Twenty-nine patients were randomized to each group. Three patients were excluded (1 due to consent error and 2 due to lack of appropriate data). Of the remaining 55 patients, 28 were assigned to the control group (with duraplasty/watertight closure) and 27 to the test group (without duraplasty/rapid-closure DC) (Fig. 4).

There was no significant difference between groups in terms of age, GCS score at the time of surgery, and GOS score (Table 2). The length of follow-up also did not differ between groups (27 vs 26.4 days, $\mathrm{p}=0.95$ ) Overall, there were complications in 9 patients, 5 in the control group and 4 in the test group, with no significant difference (Table 3). Two patients in each group developed a CSF leak. Three of them were treated conservatively with acetazolamide and/or lumbar CSF drainage, while 1 patient (in the control group) required surgery for correction. One patient in each group developed wound infection and both were treated surgically. Two patients in the control group and 1 patient in the test group developed subgaleal fluid collection seen on postoperative CT scans. These collections were probably caused by CSF leakage through duraplasty or opened dura but contained by the skin closure. Patients were asymptomatic and required no treatment.

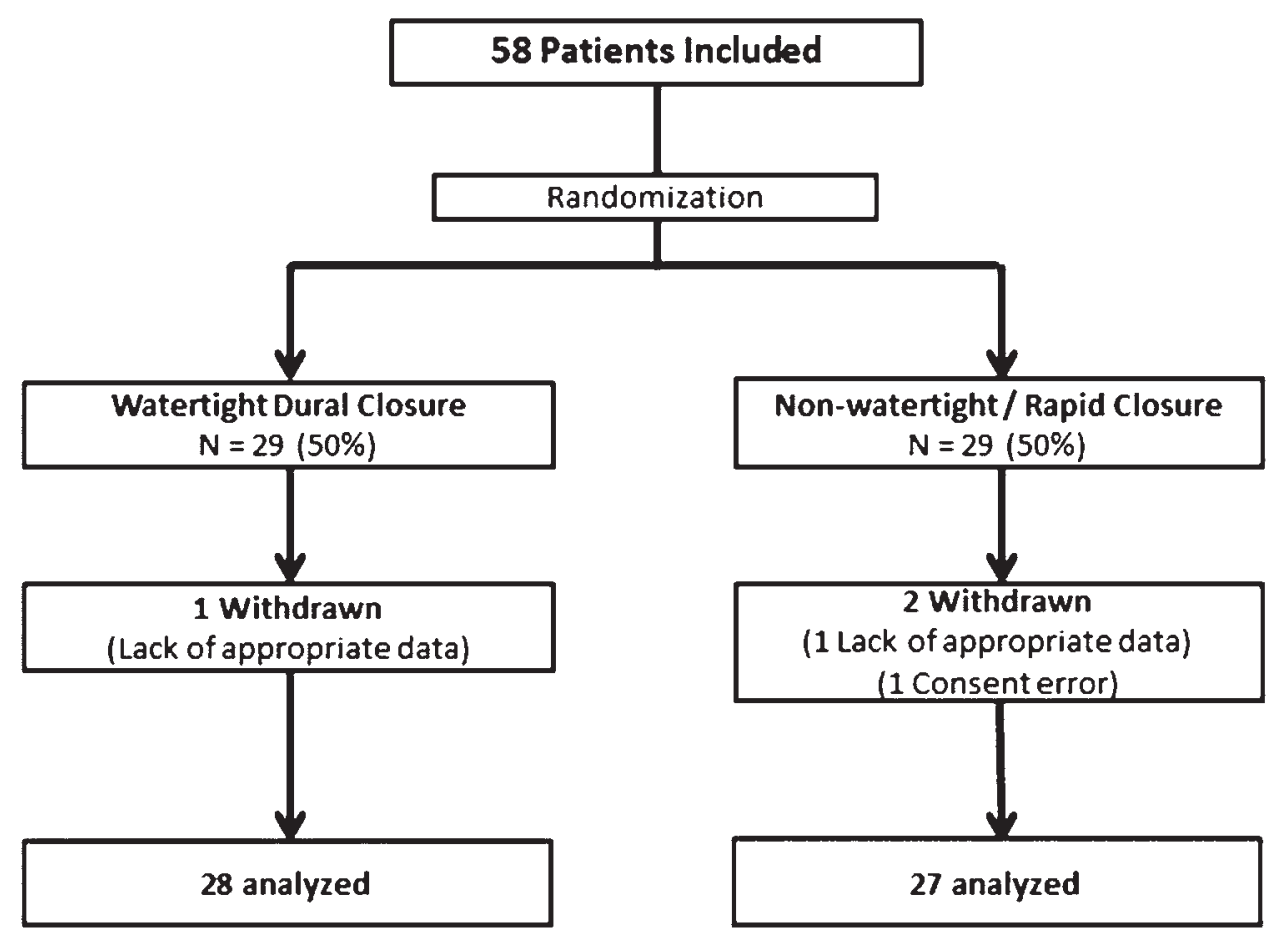

FIG. 4. Flowchart of enrollment, randomization, and follow-up of the patients. 
TABLE 2. Patient characteristics

\begin{tabular}{|c|c|c|c|}
\hline \multirow[b]{2}{*}{ Variable } & \multicolumn{2}{|c|}{ No. of Patients (\%) } & \multirow[b]{2}{*}{$\begin{array}{c}p \\
\text { Value }\end{array}$} \\
\hline & $\begin{array}{l}\text { w/ Watertight } \\
\text { Duraplasty }\end{array}$ & $\begin{array}{l}\text { w/o Watertight } \\
\text { Duraplasty }\end{array}$ & \\
\hline Total no. of patients & $28(100.0)$ & $27(100.0)$ & \\
\hline GCS score & & & $0.524^{*}$ \\
\hline $3-8$ & $14(50.0)$ & 13 (48.1) & \\
\hline $9-13$ & $12(42.9)$ & $14(51.9)$ & \\
\hline $14-15$ & $2(7.1)$ & $0(0)$ & \\
\hline Anisocoria & & & $0.130 \dagger$ \\
\hline Yes & $7(25.0)$ & $12(44.5)$ & \\
\hline No & $21(75.0)$ & $15(55.6)$ & \\
\hline GOS score & & & $0.428 \dagger$ \\
\hline 1 & $7(25.0)$ & $10(37.0)$ & \\
\hline 2 & $3(10.7)$ & $5(18.5)$ & \\
\hline 3 & $7(25.0)$ & $7(25.9)$ & \\
\hline 4 & $4(14.3)$ & $3(11.1)$ & \\
\hline 5 & $7(25.0)$ & $2(7.4)$ & \\
\hline Death & & & $0.334^{*}$ \\
\hline Yes & $7(25.0)$ & $10(37.0)$ & \\
\hline No & $21(75.0)$ & $17(63.0)$ & \\
\hline Complications & & & $1.000 \dagger$ \\
\hline Yes & $5(17.9)$ & $4(14.8)$ & \\
\hline No & $23(82.1)$ & $23(85.2)$ & \\
\hline
\end{tabular}

In the test group, the mean surgical time was 101 minutes and in the control group it was 132 minutes; the difference was 31 minutes, which was statistically significant $(\mathrm{p}=0.001)$. Considering the decrease in surgical time, operating room utilization time, anesthetic drugs, and artificial graft, there was a mean total cost reduction of $\$ 420.00$ USD (cost reduction of $23.4 \%$ ) per procedure in the test group (Table 4).

\section{Discussion}

In 1908, Cushing stated, "an accurate approximation of the dura in its two layers is desirable and should be painstakingly done!"3 This statement became the goal to be reached at the end of any case by generations of neurosurgeons aiming to prevent CSF leakage and infection. Recently, however, studies have demonstrated that a nonwatertight dural closure does not always result in a higher incidence of complications. Barth et al. ${ }^{1}$ intraoperatively randomized patients who were undergoing supratentorial craniotomies in which primary dural closure could not be accomplished into 2 groups. In the first group, watertight closure was achieved using grafts, while in the second group, the dura mater was partially sutured with 3-5 interrupted single sutures with an interspace of $2-3 \mathrm{~cm}$. Between the groups, there was no difference in the incidence of CSF leak and/or infection, and, in the nonwatertight dural closure group, surgeries were faster and less expensive (\$213 vs \$678 USD). Sade et al. ${ }^{17}$ also showed that in
TABLE 3. Incidence of complications according to randomization

\begin{tabular}{lcc}
\hline \multirow{2}{*}{ Complication } & \multicolumn{2}{c}{ No. of Patients } \\
\cline { 2 - 3 } & $\begin{array}{c}\text { w/ Watertight } \\
\text { Duraplasty }\end{array}$ & $\begin{array}{c}\text { w/o Watertight } \\
\text { Duraplasty }\end{array}$ \\
\hline CSF fistula & 2 & 2 \\
\hline Wound infection & 1 & 1 \\
\hline Subgaleal collection & 2 & 1 \\
\hline Abscess & 0 & 0 \\
\hline Total & 5 & 4 \\
\hline
\end{tabular}

surgery for supratentorial meningiomas, watertight dural closure was not superior to dural reconstruction using an artificial dural graft applied in an onlay, nonwatertight fashion.

In DC, watertight dural closure, by definition, cannot be achieved in primary fashion, i.e., solely by bringing the dural edges together. Once the decision is made to perform a watertight dural closure in a DC, some type of graft will always be needed. Such a graft can be autologous (pericranium, fascia lata) or artificial. The rationale for using such grafts is, at least theoretically, to prevent CSF leaks and infection.

Some authors, however, have proposed nonwatertight duraplasty for DC. Ragel et al., ${ }^{16}$ describing the treatment of wartime TBI during conflicts in Iraq and Afghanistan, as well as Holland and Nakaji, ${ }^{7}$ did not perform a watertight dural closure and instead utilized a dural substitute onlay graft, with no increase in the complication rates. We do not use artificial dural grafts; instead, we cover the exposed brain parenchyma with Surgicel. Güresir et al..$^{5}$ described the rapid-closure DC, a procedure that is similar to the one we used in our study. With a total of 341 procedures, the authors concluded that, compared with previously reported studies in which DC was performed with watertight dural closure, the rapid-closure technique did not increase the incidence of CSF leak, infection and/ or healing problems; in addition, surgical time was significantly decreased (69 vs 120 minutes). More recently, Kolias et al., ${ }^{14}$ in a review article, advocated the same technique for performing DC.

\section{Review of the Surgical Technique for DC}

Decompressive craniectomy is a familiar procedure for most neurosurgeons, and most of the studies focusing on its surgical technique are expert opinions or retrospective series, which creates controversy about the optimal technique. There are 2 possible cutaneous incisions through which to perform DC: the expanded reverse question mark incision and the " $\mathrm{T}$ " incision, as described by Kempe for hemispherectomy. ${ }^{9}$ The first incision begins no more than $1 \mathrm{~cm}$ anterior to the tragus and curves posteriorly around the pinna to the posterior mastoid line, where it turns superiorly, reaching the midline and then running parallel to the sagittal suture to reach the hairline. This incision provides good bone exposure but often leads to coagulation of the posterior auricular artery and occipital artery, which makes preservation of the superficial temporal ar- 
TABLE 4. Mean surgical time and costs per procedure

\begin{tabular}{ccc}
\hline Variable & Mean Op Time (mins) & Mean Cost \\
\hline w/ watertight duraplasty & 132 & $\$ 1790$ \\
\hline w/o watertight duraplasty & 101 & $\$ 1370$ \\
\hline
\end{tabular}

* In USD.

tery and its branches (frontal and parietal) crucial for an adequate skin flap vascularization and wound healing. Furthermore, healing disorders may occur on the posterior (occipital) portion of the incision due to compression by the weight of the patient's head in the supine position. The T incision consists of 2 straight incisions: a frontooccipital incision parallel to or at the midline and an incision perpendicular to the first one, starting $1 \mathrm{~cm}$ anterior to the tragus and running toward the midline until it reaches the frontooccipital incision. This incision technique may lead to injury of the parietal branch but preserves the frontal branch of the superficial temporal artery as well as the posterior auricular artery and occipital artery, leading to a better flap vascularization. It also eliminates potential healing difficulties related to compression of the wound. In our department, we often opt for the reverse question mark, with possible exceptions in elderly patients $(>60$ years old), for which we favor the $\mathrm{T}$ incision. The temporalis muscle can be elevated in a single layer along with the skin flap or separately. Our preference is to raise the skin and temporalis muscle separately because a better exposure of the squamous portion of the temporal bone can be achieved in this manner, and a better temporal lobe decompression can then be performed.

After adequate bone exposure, raising the bone flap is the next step. Jiang et al. ${ }^{8}$ carried out a randomized controlled prospective trial comparing 2 sizes of bone flaps for DC: a frontotemporoparietal flap $(12 \times 15 \mathrm{~cm})$ and a temporoparietal flap $(6 \times 8 \mathrm{~cm})$. At the 6 -month followup, $39.8 \%$ of patients in the frontotemporoparietal group had a favorable outcome according to the GOS (scores 4 and 5), compared with only $28.6 \%$ in the temporoparietal group. In addition, patients in the temporoparietal group had a higher incidence of intracranial hematomas, incisional hernias, and CSF fistulas. Since this study was published, this craniectomy size has been the standard for all DCs performed in our service. Moreover, we believe that it is important to remove temporal bone until it is flush with the middle fossa floor. Herniation of the brain parenchyma through a small craniectomy leads to compression of vessels, especially veins, against the bone edges, which makes the venous drainage difficult, exacerbating brain edema and leading to postoperative bleeding. Recently, Hartings et al. ${ }^{6}$ compared the effectiveness of the treatment of traumatic brain injury at 2 centers. In cases requiring $\mathrm{DC}$, the center in which larger bone flaps were performed $\left(82.4\right.$ vs $\left.52.4 \mathrm{~cm}^{3}\right)$ produced better clinical outcomes (67\% good results [GOS Score 4 or 5] vs $46 \%$ ). Tanrikulu et al. ${ }^{18}$ compared different sizes of DC (12-15, 15-20, and 20-24 cm) and found no additional benefit in wider craniectomies provided that the lower limit of 12 $\mathrm{cm}$ is observed.
The durotomy also must be large to avoid compression of the parenchyma against dural edges. There are several ways to perform the durotomy, each with advantages and disadvantages. In our department, the most performed durotomies are the C-shaped and the stellate durotomies. Regardless of the durotomy shape, it is important that the major vessels are not compressed against the dural edges. When this occurs, relief cuts perpendicular to the initial durotomy should be performed. Csókay et al. ${ }^{2}$ described the creation of vascular tunnels with hemostatic sponge and absorbable sutures that, at least in theory, could improve circulation to the herniated brain.

Alternative procedures for brain decompression have been proposed to replace standard DC to avoid a second surgery to replace the removed bone flap (cranioplasty). The most popular and performed procedure is the "hinge" craniotomy in which the bone flap is secured to the skull near the midline through a "Y" plate and 2 other plates are attached only to the bone flap to avoid its subsiding after brain edema resolves. ${ }^{4,12,13}$ Modifications of this technique have been proposed, such as dividing the bone flap into 2 or even 4 pieces or hinging the bone flap with sutures instead of plates, but all of them maintain the same principle of hinge. ${ }^{15,19}$ Although some studies have shown that this technique is as effective as DC, the brain expansion volume is lower (77.5 $\mathrm{ml}$ vs $105.1 \mathrm{ml})$, and in 1 study, the hinge craniotomy was associated with greater hospital mortality. $^{10,11}$ In our department, we abandoned the use of this technique due to patients requiring reoperation because of inadequate cerebral decompression, with persistent midline shift and intracranial hypertension. We believe that a possible utility for this procedure is after drainage of acute subdural hematomas in which the midline shift is smaller or equal to the thickness of the hematoma, i.e., with no important associated brain edema (Zumkeller index $\leq 0) .{ }^{20}$

\section{The Nonwatertight Duraplasty in DC (rapid-closure DC)}

We demonstrated that DC without watertight duraplasty is not associated with a higher incidence of complications when compared with the control procedure with watertight dural closure. Overall, there were 5 complications in the control group and 4 in the test group. It is important to emphasize that the evaluators in our study were blinded to randomization. The most feared complication after DC without dural closure, a CSF leak, occurred in 2 patients in the control group and only in 1 patient in the test group. Furthermore, the incidence of subgaleal fluid collections, possibly representing the occurrence of CSF leaks contained by the skin closure, was higher in the control group (2 vs 1). We concluded that once the arachnoid is intact, there is no increased risk of CSF leaks. Moreover, attempts to achieve watertight closure may lead to small defects on suture lines, causing a "one-way valve" effect that could potentially facilitate the development of CSF leakage.

Patients undergoing DC are often critically ill, especially victims of severe TBI, who are not uncommonly polytrauma patients. In these patients, faster surgical procedures focusing on the restoration of physiological parameters, known as damage control surgery (an already common concept in abdominal trauma), can be of ben- 
efit. The mean surgical time of DC in the control group was 132 minutes vs 101 minutes in the test group, i.e., the nonwatertight (rapid-closure DC) technique was 31 minutes shorter than the control procedure (this was the only statistically significant difference between all variables analyzed; $p=0.001$ ). Such a time reduction may decrease morbidity associated with longer surgeries, which are often associated with greater blood loss. This may ultimately lead to better neurosurgical outcomes.

Hospital costs were also reviewed by analyzing operating room utilization time, anesthetic drugs, and additional material (grafts) costs. The mean total costs were significantly higher in the control group. On average, there was a cost reduction of \$420 USD (23.4\% reduction) per procedure in the test group. If the rapid-closure (test) procedure had been used in all patients in our study, there would have been a total savings of $\$ 11,760$ USD.

\section{Cranioplasty}

Once it has been proven that DC without watertight duraplasty is not associated with a higher incidence of complications than the control procedure (with watertight closure), the main concern turns to cranial reconstruction surgery, in which adherence and the lack of a clear boundary between the brain parenchyma and the subcutaneous tissue could, at least theoretically, lead to an increased incidence of complications related to the cranioplasty. Our study was not powered to assess the results and complications of cranioplasty after DC without watertight duraplasty. Losses after randomization would have been too high due to deaths and patients in persistent vegetative state (who, in our practice, are not usually candidates for cranioplasty), thus precluding any significant statistical analysis. This is a point that is being addressed in an ongoing study. In our experience, cranioplasty after rapid-closure DC without watertight duraplasty (test procedure) is both feasible and safe. We usually perform cranioplasty between 6 and 12 months after DC. Once the edges of the craniotomy are found, the myocutaneous flap can be easily dissected from a neo-formed fibrous layer that covers the brain parenchyma (neo-dura) without pial injury. One should not seek to expose the parenchymal surface. This dissection itself is not far different from the one performed after DC with watertight duraplasty when pericranium is used as a graft. In such cases, a considerable amount of scarring and adherence can be found. After elevation of the myocutaneous flap, the stored bone flap or cranial implant can be fixed according to local practices. Although this process can be a little more time consuming, especially for those not accustomed to performing it, we believe that shorter operative times in critically ill patients at the expense of a few more minutes in an elective surgery (cranioplasty) is justifiable. Güresir et al., ${ }^{5}$ in a retrospective analysis of 196 cranioplasties after rapid-closure DC, found no higher incidence of complications.

\section{Limitations}

Our study is a single-center trial with a relatively small number of participants and a very specific population, which indeed diminishes the impact of our results; however, we believe that our results can help other centers and neurosurgeons to conduct new studies in different populations.

\section{Conclusions}

In our study, DC without watertight duraplasty was not associated with a higher incidence of postoperative complications (i.e., CSF leaks, wound infections, abscesses, and subgaleal collections) and decreased surgical time by 31 minutes on average, which can be beneficial in critically ill patients, especially in victims of severe TBI. We also noted a reduction in hospital costs (\$420 USD [a 23.4\% reduction] per patient on average). Our study is a randomized controlled trial addressing the surgical technique for DC, a widely used procedure in neurosurgical practice; it provides data for the rational choice of the best technique to be employed in each case.

\section{References}

1. Barth M, Tuettenberg J, Thomé C, Weiss C, Vajkoczy P, Schmiedek P: Watertight dural closure: is it necessary? A prospective randomized trial in patients with supratentorial craniotomies. Neurosurgery 63 (4 Suppl 2):352-358, 2008

2. Csókay A, Együd L, Nagy L, Pataki G: Vascular tunnel creation to improve the efficacy of decompressive craniotomy in post-traumatic cerebral edema and ischemic stroke. Surg Neurol 57:126-129, 2002

3. Cushing H: Surgery of the head, in Keen WW (ed): Surgery, Its Principles and Practice, vol III. Philadelphia: Saunders, 1908, pp 17-276

4. Goettler CE, Tucci KA: Decreasing the morbidity of decompressive craniectomy: the Tucci flap. J Trauma 62:777-778, 2007

5. Güresir E, Vatter H, Schuss P, Oszvald A, Raabe A, Seifert V, et al: Rapid closure technique in decompressive craniectomy. J Neurosurg 114:954-960, 2011

6. Hartings JA, Vidgeon S, Strong AJ, Zacko C, Vagal A, Andaluz N, et al: Surgical management of traumatic brain injury: a comparative-effectiveness study of 2 centers. J Neurosurg 120:434-446, 2014

7. Holland M, Nakaji P: Craniectomy: surgical indications and technique. Oper Tech Neurosurg 7:10-15, 2004

8. Jiang JY, Xu W, Li WP, Xu WH, Zhang J, Bao YH, et al: Efficacy of standard trauma craniectomy for refractory intracranial hypertension with severe traumatic brain injury: a multicenter, prospective, randomized controlled study. $\mathbf{J}$ Neurotrauma 22:623-628, 2005

9. Kempe LK: Hemispherectomy, in Salcman M, Heros RC, Laws E, et al (eds): Kempe's Operative Neurosurgery, ed 2. New York: Springer, 2004, pp 170-176

10. Kenning TJ, Gandhi RH, German JW: A comparison of hinge craniotomy and decompressive craniectomy for the treatment of malignant intracranial hypertension: early clinical and radiographic analysis. Neurosurg Focus 26(6):E6, 2009

11. Kenning TJ, Gooch MR, Gandhi RH, Shaikh MP, Boulos AS, German JW: Cranial decompression for the treatment of malignant intracranial hypertension after ischemic cerebral infarction: decompressive craniectomy and hinge craniotomy. J Neurosurg 116:1289-1298, 2012

12. Khoo JC: Replacement of a self-adjusting bone flap. J Neurosurg 45:589-591, 1976

13. Ko K, Segan S: In situ hinge craniectomy. Neurosurgery 60 (4 Suppl 2):255-259, 2007

14. Kolias AG, Kirkpatrick PJ, Hutchinson PJ: Decompressive craniectomy: past, present and future. Nat Rev Neurol 9:405-415, 2013 
15. Peethambaran AK, Valsalmony J: Four-quadrant osteoplastic decompressive craniotomy: a novel technique for decompressive craniectomy avoiding revision cranioplasty after surgery. Neurol India 60:672-674, 2012

16. Ragel BT, Klimo P Jr, Martin JE, Teff RJ, Bakken HE, Armonda RA: Wartime decompressive craniectomy: technique and lessons learned. Neurosurg Focus 28(5):E2, 2010

17. Sade B, Oya S, Lee JH: Non-watertight dural reconstruction in meningioma surgery: results in 439 consecutive patients and a review of the literature. J Neurosurg 114:714-718, 2011

18. Tanrikulu L, Oez-Tanrikulu A, Weiss C, Scholz T, Schiefer J, Clusmann H, et al: The bigger, the better? About the size of decompressive hemicraniectomies. Clin Neurol Neurosurg 135:15-21, 2015

19. Valença MM, Martins C, da Silva JC: "In-window" craniotomy and "bridgelike" duraplasty: an alternative to decompressive hemicraniectomy. J Neurosurg 113:982-989, 2010

20. Zumkeller M, Behrmann R, Heissler HE, Dietz H: Computed tomographic criteria and survival rate for patients with acute subdural hematoma. Neurosurgery 39:708-713, 1996

\section{Disclosures}

The authors report no conflict of interest concerning the materials or methods used in this study or the findings specified in this paper.

\section{Author Contributions}

Conception and design: Vieira, Faquini, Almeida, AzevedoFilho. Acquisition of data: Vieira, Guimarães, Faquini, Silva, Oliveira, Andrade, Gemir, Neri. Analysis and interpretation of data: Vieira, Faquini, Oliveira, Azevedo-Filho. Drafting the article: Vieira, Azevedo-Filho. Critically revising the article: Vieira, Guimarães, Faquini, Almeida, Azevedo-Filho. Reviewed submitted version of manuscript: Vieira, Almeida, AzevedoFilho. Approved the final version of the manuscript on behalf of all authors: Vieira. Statistical analysis: Vieira, Silva. Study supervision: Vieira.

\section{Correspondence}

Eduardo Vieira, Department of Neurological Surgery, Hospital da Restauração, Avenida Agamenon Magalhães SN, Recife PE 52010040, Brazil. email: evcj2005@gmail.com. 